INRA Prod. Anim., 1991, 4 (4), $287-295$

\author{
A. MULLER, D. MICOL*, \\ J.R. PECCATTE, D. DOZIAS \\ INRA \\ Domaine du Pin au Haras \\ 61310 Exmes \\ * INRA \\ Laboratoire Croissance \\ et Métabolisme des Herbivores \\ Theix - 63122 Saint-Genès-Champanelle
}

\section{Choix de l'âge à la castration en production de viande bovine semi-intensive}

La production intensive de viande bovine a montré l'intérêt du mâle entier. La castration des animaux à l'herbe étant nécessaire, on a souvent recommandé de l'effectuer tardivement. Cependant cette opération ne doit pas être trop retardée au risque de pénaliser l'animal, sans pour autant obtenir de meilleurs résultats.

Lorsque le système de production de viande bovine est basé sur l'utilisation maximum du pâturage, il est inévitable de castrer les jeunes mâles. Dans le cas des races à viande spécialisées où la naissance a lieu en fin d'hiver, la castration se fait traditionnellement vers 5-7 mois, lors de la rentrée en stabulation. L'intensification de la production à partir de jeunes bovins a incité à repousser la castration à un âge ultérieur, afin de faire bénéficier plus longtemps l'animal de la supériorité de croissance du mâle entier (Seideman et al 1982, Micol 1986) mais l'opération doit rester possible à la pince, et sans stress excessif pour l'animal (Nocquet 1977). Dans la pratique, les broutards peuvent

\section{Résumé}

La production de bovins à viande à partir de ressources herbagères utilise essentiellement des animaux mâles castrés. Le fait que l'animal entier présente un potentiel de croissance supérieur a conduit à recommander, pour cette production, un âge à la castration tardif au risque de pénaliser fortement l'animal, lors de cette opération, s'il atteint un développement déjà important.

Le but des études conduites au Domaine INRA du Pin au Haras a été de préciser les effets d'une castration à 9 ou à 13 mois sur la production de boeufs à partir d'herbe. Ce facteur a été étudié en interrelation avec d'autres facteurs d'élevage au cours de trois essais consécutifs : précocité des génotypes des animaux, niveau d'alimentation au cours de l'hiver, âge à l'abattage.

Il ressort globalement que l'âge à la castration dans la plage étudiée modifie assez peu les caractéristiques finales des animaux. De nombreuses interactions avec les autres facteurs étudiés et des compensations entre les diverses périodes de la vie productive semblent réduire les effets attendus de l'âge à la castration. Cependant les carcasses des animaux castrés les plus tard semblent présenter un état d'engraissement moindre à l'abattage.

En définitive, au sein des élevages, le choix de l'âge à la castration aux environs d'un an dépendra surtout de la faisabilité et de la pénibilité de l'opération pour l'animal. donc être castrés soit à l'automne, vers $7-9$ mois d'âge, lorsqu'ils pèsent 250 à $320 \mathrm{~kg}$, soit un peu avant la fin de l'hiver, vers 12-13 mois, à un poids de $350-420 \mathrm{~kg}$, voire un peu plus. Des études antérieures ont même précisé les effets d'âge à la castration très tardif (18 mois), sans retenir leur intérêt pratique compte tenu des contre performances observées à ce poids et à ce stade de développement de l'animal lors de la castration (Micol 1986, Robelin et Boccard 1978).

Il n'est pas certain que l'avantage de poids habituellement acquis chez l'animal entier pendant le premier hiver soit conservé ensuite au pâturage, puis jusqu'à l'abattage. Aussi trois essais comparant deux âges à la castration ( $\mathbf{9}$ et 13 mois) ont été réalisés de 1985 à 1988 au Domaine INRA du Pin au Haras, avec des animaux plus ou moins précoces d'origine allaitante ou laitière, conduits selon différents niveaux d'alimentation pendant le premier hiver, avant d'être remis au pâturage puis engraissés pour être abattus soit à 24 , soit à 30 mois, comme cela se fait classiquement (Micol et Béranger 1984).

Les schémas expérimentaux de ces trois essais sont présentés en annexe. La conduite expérimentale se résume ainsi. Les animaux, nés en moyenne fin février, ont pâturé le premier été soit avec leurs mères pour les animaux de race à viande soit après un sevrage précoce pour les animaux laitiers. Ils ont été regroupés début novembre dans une stabulation libre paillée par lots de 12 ou 13 et ont reçu à volonté durant l'hiver une ration à base d'ensilage de maïs immature ou de ray gras anglais complémentée par des minéraux et par 0,5 à $1 \mathrm{~kg}$ de concentré, plus ou moins riche en azote suivant la composition de l'ensilage ( $\mathrm{cf}$ annexe). 
Après 3 ou 4 semaines d'adaptation au régime de fourrages conservés, ces animaux ont été répartis par races en 2 lots comparables sur la base de l'âge et du poids. L'un des lots a été aussitôt castré ( "castrés à 9 mois »), l'autre ( "castrés à 13 mois ») a subi la même opération à la mi-mars, 3 à 5 semaines avant la mise à l'herbe. De la mise en lot à la mise à l'herbe, on a mesuré par lot les quantités d'ensilage offertes et refusées 4 jours par semaine. Les animaux ont été pesés (simple ou double pesée selon le cas) toutes les 3 semaines environ au cours de l'hiver. Les mesures effectuées sur les ensilages ont porté sur la teneur en matière sèche et sa composition (MM, MAT, CB), la

Figure 1. Evolution du poids vif des animaux entre 9 et 20 mois selon l'âge à la castration et le génotype (essai 1).

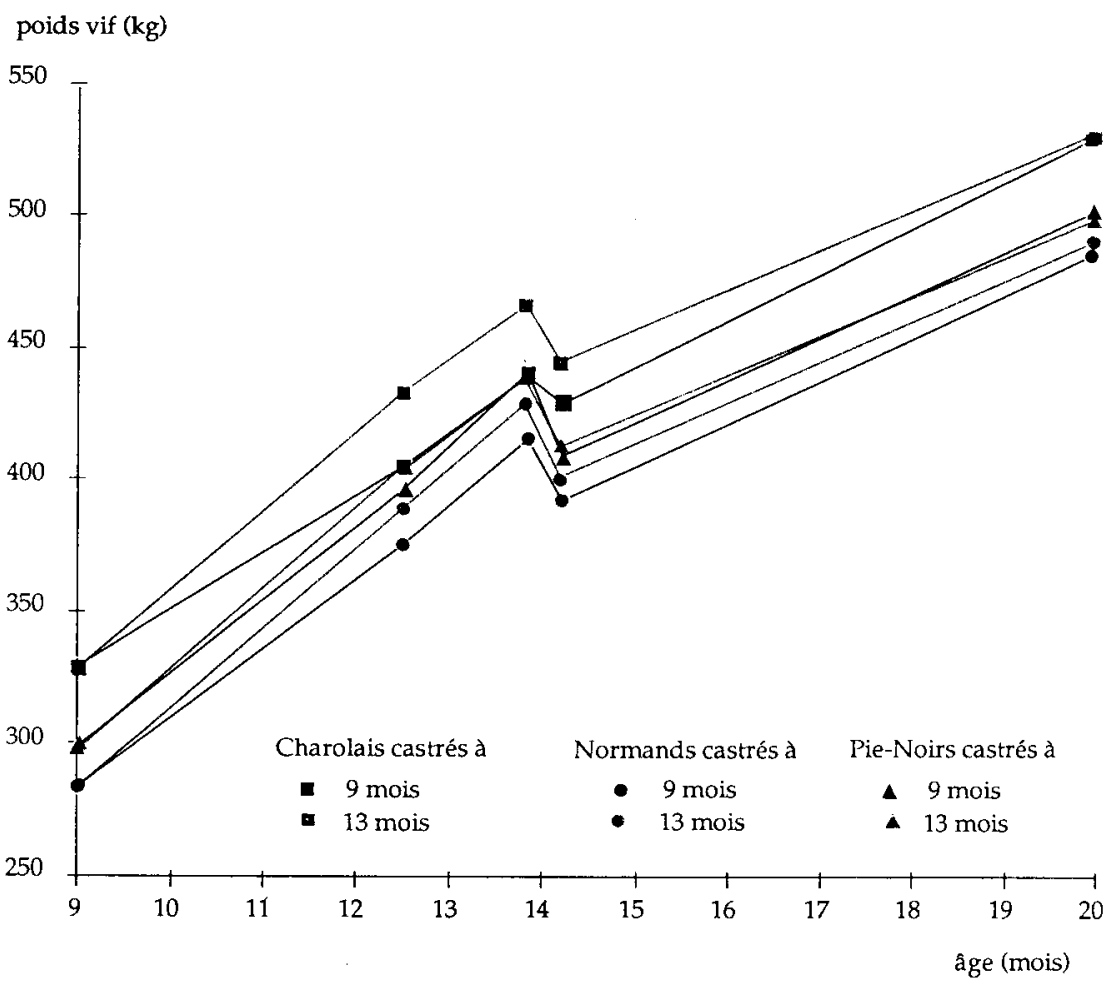

digestibilité sur moutons et les caractéristiques fermentaires.

Pendant le second été, entre les âges de 14 et 20 mois, les animaux castrés à 9 et à 13 mois ont été conduits ensemble au pâturage, les charolais généralement à part des animaux d'origine laitière. Ils ont été pesés environ toutes les 4 semaines jusqu'à la fin du pâturage. La conduite des animaux au delà de 20 mois a été différente selon les trois essais et est précisée ultérieurement pour chacun d'eux.

\section{1 / Effets de l'âge à la castration selon la race (Essai 1)}

Nous avons disposé dans cette expérience de 24 ou 26 jeunes animaux de chacune des 3 races charolaise, normande et pie-noire $\mathrm{x}$ holstein.

Les animaux castrés à 9 mois étaient âgés en moyenne de 271 jours et pesaient $305 \mathrm{~kg}$; les autres animaux ont été castrés à 13 mois, 110 jours plus tard, au poids moyen de $410 \mathrm{~kg}$ après avoir réalisé des gains de poids comparables entre races (tableau 1).

\section{1 / Résultats}

De la mise en lot à la mise à l'herbe (20 semaines), les animaux castrés à 13 mois ont consommé plus d'ensilage que les animaux castrés à 9 mois, $+9 \%$ pour les charolais et + $11 \%$ pour les normands (tableau 1). Les différences sont atténuées (respectiventent $+5 \%$ et $+9 \%$ lorsqu'on rapporte les quantités consommées au poids moyen hivernal, du fait des croîts plus faibles des animaux castrés à 9 mois : - $190 \mathrm{~g} / \mathrm{j}$ pour les charolais, - $90 \mathrm{~g} / \mathrm{j}$ pour les normands. En race pie-noire, il n'y a pas eu d'écart significatif entre les 2 lots, ni pour les quantités consommées ni pour les croissances.

Par ailleurs, on n'a constaté aucune baisse importante de l'appétit dans les 2 ou 3

\footnotetext{
Tableau 1. Poids vifs, croissances et consommations selon la race et l'âge à la castration (Essai 1).
}

\begin{tabular}{|c|c|c|c|c|c|c|}
\hline Race & \multicolumn{2}{|c|}{ Charolaise } & \multicolumn{2}{|c|}{ Normande } & \multicolumn{2}{|c|}{ Pie-Noire } \\
\hline Age à la castration (mois) & 9 & 13 & 9 & 13 & 9 & 13 \\
\hline $\begin{array}{l}\text { Périoóde de } 9 \text { à } 14 \text { mois } \\
\text { Consommation } \\
\text { d'ensilage }(\mathrm{kg} \mathrm{MS} / \mathrm{j}) \\
\text { Consommation } \\
\text { d'ensilage }(\mathrm{kg} \text { MS } / 100 \mathrm{~kg} \\
\text { de poids vif) } \\
\text { Poids à la castration } \\
\text { (kg) } \\
\text { Croissance }(\mathrm{g} / \mathrm{j}) \\
\text { sur } 146 \mathrm{j} \\
\text { Gain de poids vif } \\
\text { en hiver (kg) }\end{array}$ & $\begin{array}{c}5,86 \\
1,51 \\
330 \pm 41 \\
752 \mathrm{a} \pm 152 \\
110 \pm 22\end{array}$ & $\begin{array}{c}6,36 \\
1,59 \\
433 \pm 45 \\
941 b \pm 65 \\
138 \pm 9\end{array}$ & $\begin{array}{c}5,76 \\
1,65 \\
284 \pm 42 \\
900 \mathrm{a} \pm 92 \\
132 \pm 13\end{array}$ & $\begin{array}{c}6,38 \\
1,80 \\
389 \pm 43 \\
991 a \pm 137 \\
145 \pm 20\end{array}$ & $\begin{array}{c}7,27 \\
1,96 \\
299 \pm 36 \\
978 a \pm 73 \\
143 \pm 11\end{array}$ & $\begin{array}{c}7,03 \\
1,90 \\
405 \pm 37 \\
959 a \pm 88 \\
140 \pm 13\end{array}$ \\
\hline $\begin{array}{l}\text { Période de } 9 \text { à } 20 \text { mois } \\
\text { Gain total de poids vif } \\
(\mathrm{kg}) \text { sur } 332 \mathrm{j}\end{array}$ & $197 \pm 36$ & $201 \pm 36$ & $202 \pm 19$ & $206 \pm 17$ & $203 \pm 24$ & $199 \pm 26$ \\
\hline
\end{tabular}

Pour une même race, les valeurs suivies de lettres différents sont significativement différentes $(\mathrm{P}<0,05)$. 


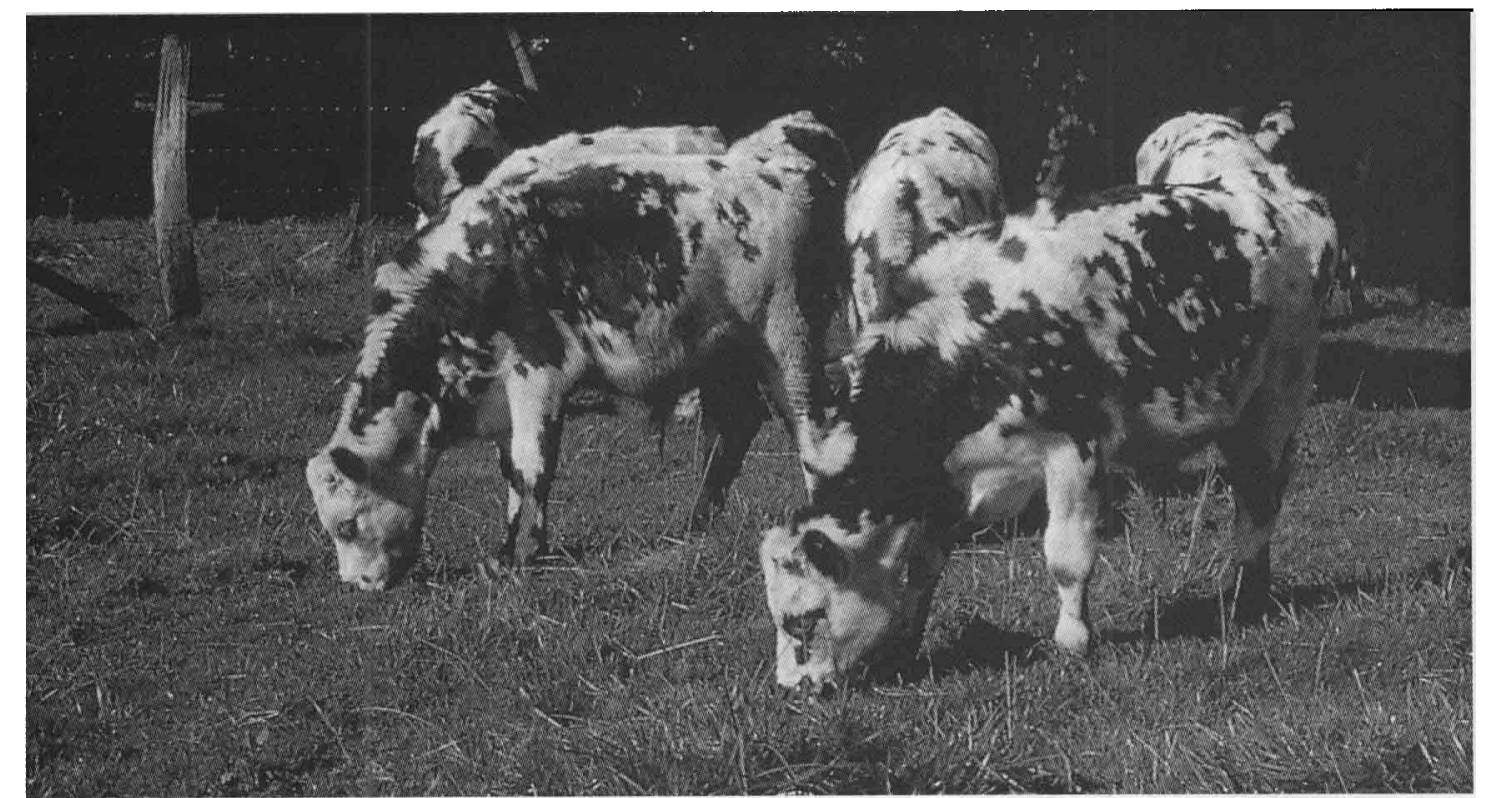

semaines suivant la castration quel que soit l'âge où elle a été pratiquée et quelle que soit la race.

Globalement, les gains de poids réalisés en hiver entre 9 et 14 mois ont peu varié entre races dans le cas de la castration tardive (138 à $145 \mathrm{~kg}$ ). Par rapport à ces gains de poids, ceux des animaux castrés à $\mathbf{9}$ mois ont été respectivement de 80,91 et $102 \%$ pour les charolais, les normands et les pie-noirs (tableau 1). Les écarts de poids des charolais et des normands se sont progressivement comblés au pâturage, d'abord à la mise à l'herbe (les castrés à 13 mois plus lourds perdent alors plus de poids) puis en cours d'été, alors que les poids des pienoirs des 2 lots sont toujours restés très proches (figure 1).

En fin d'automne, et quelle que soit la race, les effets de l'âge à la castration paraissent annulés. Les gains de poids totaux au cours de l'hiver et de l'été sont par ailleurs peu différents entre races : 198, 204 et $201 \mathrm{~kg}$ respectivement pour charolais, normands et pie-noirs (figure 1).

Au cours de l'hiver suivant de 20 à 24 mois, les animaux castrés à 13 mois ont reçu une ration correspondant à un haut niveau d'alimentation et ceux castrés à 9 mois à un bas niveau d'alimentation, puis ils ont tous été engraissés à l'herbe à 30 mois. De ce fait, l'effet strict de l'âge à la castration ne peut être étudié que jusqu'à 20 mois.

\section{2 / Discussion}

La castration à 9 mois a d'autant plus réduit les consommations de fourrage et les croîts hivernaux des animaux que ceux-ci avaient une aptitude à la production de viande plus marquée et une moindre précocité. Cependant, lors de cette opération, les charolais étaient nettement plus lourds que les normands ( $+45 \mathrm{~kg}$ ) et même que les pie-noirs ( $+30 \mathrm{~kg}$ ), donc peutêtre relativement plus en avance dans leur développement corporel. La castration a réduit d'autant plus les croissances qu'elle a lieu plus tôt et s'applique à des animaux ayant une forte capacité de croissance musculaire. Cependant dans des conditions d'alimentation favorables les animaux sont capables de combler leur retard de croissance. En effet, si l'on rapporte le gain de poids à l'herbe des animaux castrés à 13 mois à celui des animaux castrés à 9 mois, il est de 72,87 et $98 \%$ respectivement pour les charolais, normands et pie-noirs.

On peut regretter que l'essai n'ait pu continuer au delà des 20 mois car il n'est pas exclu que durant les périodes suivantes (hiver et pâturage) il y ait eu aussi un phénomène de croissance compensatrice.

\section{2 / Effets de l'âge à la castration selon le niveau alimentaire (Essai 2)}

Durant le premier hiver (9-14 mois d'âge), les charolais et les pie-noirs ont reçu ou non un apport de concentré (lot $\mathrm{HN}=+1,5 \mathrm{~kg}$ ) (lot BN $=0$ ), en supplément de la ration de fourrages complémentée en azote. Lors de la mise en lot avant la castration à 9 mois, les paramètres pris en compte pour la répartition des charolais et des pie-noirs ont été l'âge et le poids. Cependant dans le cas des pie-noirs, il a fallu tenir compte du passé des animaux. Ceux-ci avaient été déjà conduits selon deux niveaux de croissance pendant la période de pâturage précédente après le sevrage ce qui avait entraîné un écart de poids de $34 \mathrm{~kg}$ entre les deux niveaux. Cette répartition des pie-noirs a été maintenue pour la suite et jusqu'à l'abattage et c'est à l'intérieur de ces deux lots initiaux que la répartition entre animaux castrés aussitôt ou en fin d’hiver a été réalisée.

Les animaux ont reçu pendant le second hiver (soit de 20 à 24 mois) une ration de foin
Pour les 3 races l'âge à la castration paraît sans effet sur le gain total de poids à 20 mois. 
ou d'ensilage, de faible qualité, avec dans chaque lot des animaux castrés tôt et tard mélangés. Ils ont tous été engraissés ensuite au pâturage, avec une complémentation de $3 \mathrm{~kg}$ de concentré pendant 70 jours dans le cas des charolais.

L'âge moyen des animaux lors de la première opération de castration, peu différent entre

Figure 2. Evolution du poids vif des animaux entre 9 et 30 mois selon l'âge à la castration (essai 2).

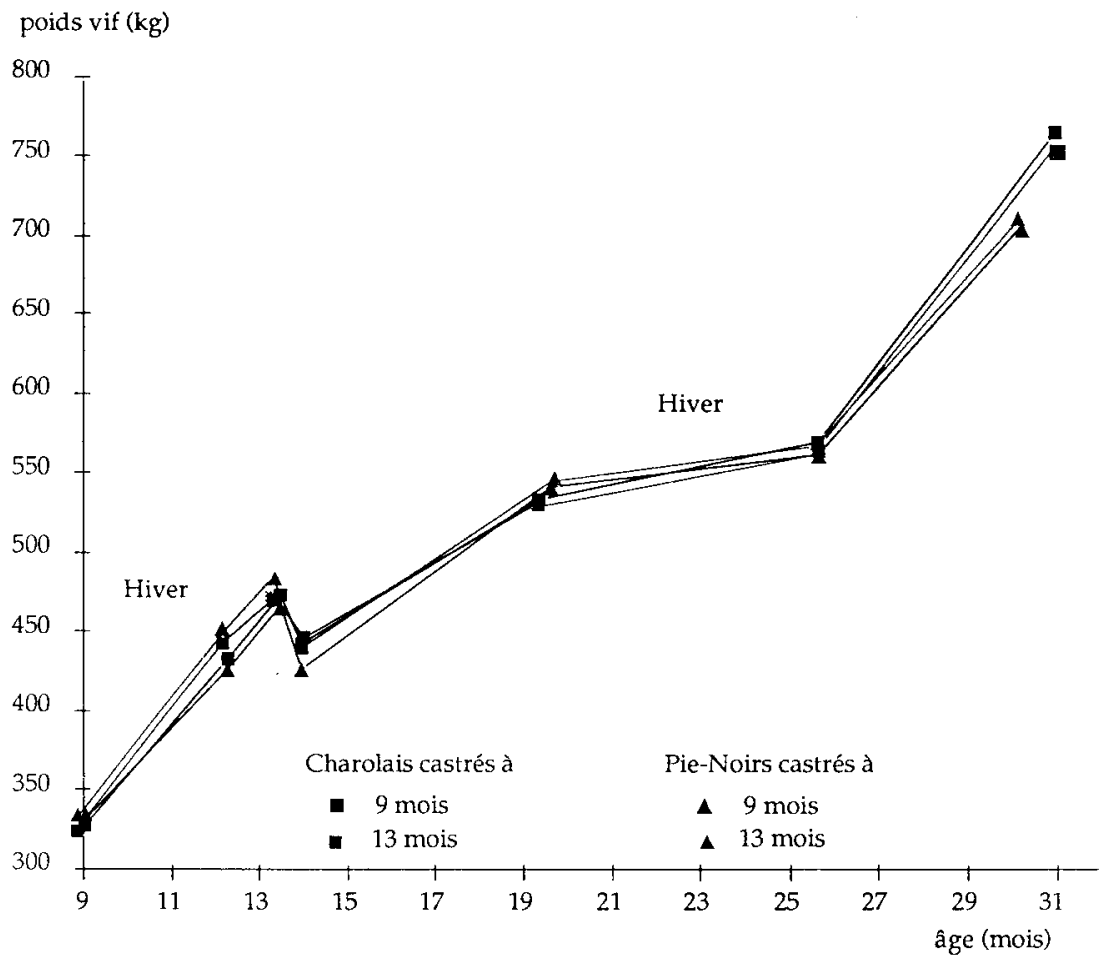

races, était de 295 j soit presque 10 mois. Le poids moyen était sensiblement le même pour les 2 races : $327 \mathrm{~kg}$ chez les charolais, $332 \mathrm{~kg}$ chez les pie-noirs. Les animaux castrés à 13 mois ont subi l'opération $105 \mathrm{j}$ plus tard, à un poids de $447 \mathrm{~kg}$ et de $457 \mathrm{~kg}$ respectivement pour les charolais et les pie-noirs.

\section{1 / Résultats}

En race charolaise ( $\mathrm{HN}$ et $\mathrm{BN}$ ), ni les quantités ingérées ni les croîts sur l'ensemble de l'hiver n'ont été sensiblement affectés par l'âge à la castration (tableau 2). La différence de croissance entre castrés à 9 mois et à 13 mois a été pratiquement comblée avant la mise à l'herbe. En race pie-noire, les animaux castrés à 9 mois ont ingéré un peu moins d'ensilage (de 0,3 à $0,4 \mathrm{~kg}$ de MS) et ont réalisé des croîts nettement plus faibles (- $160 \mathrm{~g}$ soit $14 \%$ ) que les animaux castrés à 13 mois (figure 2 ).

Dans les deux races, les lots HN ont ingéré des rations totales augmentées de 13 à $14 \%$ par l'apport de concentré. En race charolaise, l'effet de cet apport sur le croît est deux fois plus élevé (150 g) qu'en race pie-noire (65 g). Mais globalement le concentré a plutôt amélioré l'état corporel que les croîts en fin d'hiver.

Ainsi, au cours du premier hiver (9-14 mois), l'effet de l'âge à la castration en race tardive, constaté dans l'essai 1, n'est pas confirmé, alors qu'il est net cette fois en race précoce, mais sans interaction avec le niveau d'alimentation.

Entre 14 et 20 mois, l'effet de la mise à l'herbe puis des croissances réalisées au pâturage ont compensé les écarts de poids des différents lots par race. Ainsi de 9 à 20 mois au total, les gains de poids des charolais castrés tôt et tard sont respectivement de 208 et $214 \mathrm{~kg}$; ceux des pie-noirs, de 210 et $215 \mathrm{~kg}$. Un léger

Tableau 2. Poids vifs, croissances et consommations selon le niveau d'alimentation, la race et l'âge à la castration (Essai 2).

\begin{tabular}{|c|c|c|c|c|c|c|c|c|}
\hline Race & \multicolumn{4}{|c|}{ Charolaise } & \multicolumn{4}{|c|}{ Pie-Noire } \\
\hline Niveau d'alimentation & \multicolumn{2}{|c|}{ Bas } & \multicolumn{2}{|c|}{ Haut } & \multicolumn{2}{|c|}{ Bas } & \multicolumn{2}{|c|}{ Haut } \\
\hline $\begin{array}{l}\text { Période de } 9 \text { à } 14 \text { mois } \\
\text { Consommation } \\
\text { d'ensilage }(\mathrm{kg} \mathrm{MS} / \mathrm{j}) \\
\text { Consommation } \\
\text { d'ensilage }(\mathrm{kgMS} / \mathbf{1 0 0 ~ k g} \\
\text { de poids vif) } \\
\text { Poids vif } \\
\text { à la castration }(\mathrm{kg}) \\
\text { Croissance } \\
\text { journalière }(\mathrm{g} / \mathrm{j})(135 \mathrm{j})\end{array}$ & $\begin{array}{c}6,03 \\
1,68 \\
323 \pm 34 \\
1004 a \pm 161\end{array}$ & $\begin{array}{c}6,06 \\
1,68 \\
433 \pm 41 \\
1076 a \pm 157\end{array}$ & $\begin{array}{c}1,84 \\
331 \pm 30 \\
1172 b \pm 179\end{array}$ & $\begin{array}{c}5,89 \\
1,86 \\
462 \pm 32 \\
1204 \mathrm{~b} \pm 137\end{array}$ & $\begin{array}{c}6,77 \\
1,89 \\
315 \pm 27 \\
975 a \pm 122\end{array}$ & $\begin{array}{c}7,18 \\
1,97 \\
431 \pm 37 \\
1111 b \pm 153\end{array}$ & $\begin{array}{c}1,97 \\
350 \pm 27 \\
1014 \mathrm{a} \pm 91\end{array}$ & $\begin{array}{c}6,88 \\
1,98 \\
482 \pm 51 \\
1201 b \pm 133\end{array}$ \\
\hline
\end{tabular}

Par race, les croissances suivies de lettres différentes sont significativement différentes $(\mathrm{P}<0,01)$ et les poids suivis de lettres différentes sont significativement différents $(\mathrm{P}<0,05)$. 
Tableau 3. Résultats d'engraissement et d'abattage selon le niveau d'alimentation, la race et l'âge à la castration (Essai 2).

\begin{tabular}{|c|c|c|c|c|c|c|c|c|}
\hline Race & \multicolumn{4}{|c|}{ Charolaise } & \multicolumn{4}{|c|}{ Pie-Noire } \\
\hline Niveau d'alimentation & \multicolumn{2}{|c|}{ Bas } & \multicolumn{2}{|c|}{ Haut } & \multicolumn{2}{|c|}{ Bas } & \multicolumn{2}{|c|}{ Haut } \\
\hline $\begin{array}{l}\text { Age à la castration } \\
\text { (mois) }\end{array}$ & 9 & 13 & 9 & 13 & 9 & 13 & 9 & 13 \\
\hline $\begin{array}{l}\text { Période de finition } \\
\text { à l'herbe } \\
\text { Durée de finition (j) } \\
\text { Croissance } \\
\text { journalière (g/j) } \\
\text { Poids vif départ } \\
\text { à l'abattoir (kg) }\end{array}$ & $\begin{array}{c}162 \\
1185 a \pm 213 \\
752\end{array}$ & $\begin{array}{c}162 \\
1152 a \pm 190 \\
768\end{array}$ & $\begin{array}{c}162 \\
1229 a \pm 160 \\
781\end{array}$ & $\begin{array}{c}162 \\
1192 a \pm 145 \\
748\end{array}$ & $\begin{array}{c}153 \\
1037 a \pm 75 \\
702\end{array}$ & $\begin{array}{c}153 \\
1029 a \pm 246 \\
708\end{array}$ & $\begin{array}{c}122 \\
1103 a \pm 205 \\
713\end{array}$ & $\begin{array}{c}122 \\
1099 a \pm 143 \\
718\end{array}$ \\
\hline $\begin{array}{l}\text { Abattage } \\
\text { Poids de carcasse } \\
\text { froide (kg) } \\
\text { Dépôts adipeux } \\
\text { en \% du poids } \\
\text { de carcasse }\end{array}$ & $426 a \pm 22$ & $438 a \pm 25$ & $439 a \pm 27$ & $416 a \pm 25$ & $\begin{array}{l}354 a \pm 16 \\
16,0 a \pm 1,3\end{array}$ & $\begin{array}{c}355 a \pm 21 \\
14,1 b \pm 1,7\end{array}$ & $\begin{array}{l}359 a \pm 18 \\
16,0 a \pm 1,6\end{array}$ & $\begin{array}{l}360 \mathrm{a} \pm 20 \\
15,6 \mathrm{a} \pm 1,6\end{array}$ \\
\hline
\end{tabular}

Par race, les valeurs suivies de lettres différenles sont significativement différentes $(\mathrm{P}<0,05)$.

avantage demeure donc en faveur de la castration tardive. Dans le cas des charolais cependant ces moyennes cachent une certaine disparité ; en fin de pâturage, les castrés à 13 mois sont plus lourds de $16 \mathrm{~kg}$ pour le lot $\mathrm{BN}$, plus légers de $13 \mathrm{~kg}$ pour le lot $\mathrm{HN}$, que les castrés à 9 mois.

Entre l'automne au pâturage et la pesée après la mise à l'herbe suivante à 25 mois, les gains ont été faibles mais comparables (20 à $27 \mathrm{~kg}$ ) entre les lots de pie-noirs; ils ont été variables (21 à $45 \mathrm{~kg}$ ) chez les charolais et ont accentué la disparité constatée à 20 mois. En effet, les animaux castrés à 13 mois sont dès lors plus lourds de $22 \mathrm{~kg}$ pour le lot $\mathrm{BN}$, plus légers de $27 \mathrm{~kg}$ pour le lot HN. L'analyse fait ressortir dans cette race une interaction significative $(\mathrm{P}<0,05)$ entre niveau d'alimentation et âge à la castration.

\section{2 / Engraissement et abattage des Charolais}

Les croîts des 4 lots de charolais engraissés à l'herbe (tableau 3) ont peu varié autour d'une moyenne de $1190 \mathrm{~g} / \mathrm{j}$, en partie sans doute par l'effet de la complémentation en aliments concentrés (3 kg à partir du 15 juillet). Les animaux castrés à 9 mois ont réalisé $35 \mathrm{~g} / \mathrm{j}$ de plus que les animaux castrés à 13 mois, et les animaux du lot HN $40 \mathrm{~g} / \mathrm{j}$ de plus que ceux du lot $B N$. Les durées de finition entre animaux ont varié plus largement de 137 à $194 \mathrm{j}$. Ainsi, pour une durée moyenne de $162 \mathrm{j}$, les gains de poids ont varié de 186 à $199 \mathrm{~kg}$ selon les lots, et les lots les plus légers à la mise à l'herbe (BN castrés tôt et HN castrés tard) sont restés les plus légers en fin de pâturage.

L'abattage a eu lieu à GACE (61) où il n'a pas été possible d'obtenir d'autres données que le poids de carcasse (sans émoussage). Le rendement en carcasse a été plus élevé pour les lots BN que pour les lots $H N$, de $0,5 \%$ pour les animaux castrés à 9 mois, de $1,5 \%$ pour les animaux castrés à 13 mois. Ainsi les poids de carcasse les plus élevés sont obtenus par les BN castrés tard et les HN castrés tôt (438 et $439 \mathrm{~kg}$ respectivement). Le lot HN castré à 13 mois, qui est resté le lot le plus léger depuis la fin de l'été précédent et qui a le plus mauvais rendement, donne les carcasses les plus légères (416 kg).

\section{3 / Engraissement et abattage des Pie-Noirs}

Le croît moyen des 4 lots de pie-noirs a été de $1070 \mathrm{~g} / \mathrm{j}$ pendant la finition à l'herbe (tableau 3), sans variation notable selon l'âge à la castration de sorte que l'écart de poids de $7 \mathrm{~kg}$ des castrés tôt à la mise à l'herbe se maintient en finition $(708 \mathrm{~kg}$ pour les animaux castrés à 9 mois, contre $713 \mathrm{~kg}$ pour ceux castrés à 13 mois)

Pour compenser leur plus faible poids créé dans la période $5-9$ mois (- $34 \mathrm{~kg}$ ) et conservé jusqu'à 25 mois, les animaux du lot $\mathrm{BN}$ ont bénéficié d'une finition à l'herbe de $31 \mathrm{j}$ plus longue. Ceci n'a pas suffi pour leur permettre d'atteindre le même poids que le lot $\mathrm{HN}$ (705 kg, contre $715 \mathrm{~kg}$ respectivement lors du départ à l'abattoir). En effet la valeur alimentaire de l'herbe diminue en cours d'été et le croît a été nettement limité en fin d'engraissement à l'herbe sans complémentation.

Lors de l'abattage (à l'INRA de Theix), on n'a pas constaté de différence de poids de carcasse entre animaux castrés à 9 mois $(357 \mathrm{~kg})$ et à 13 mois $(358 \mathrm{~kg})$. Par contre, l'état d'engraissement, exprimé par la proportion de dépôts adipeux totaux de la carcasse, a été significative-
La castration tardive des Charolais, associée à un haut niveau alimentaire, a conduit aux poids de carcasse les plus faibles. 
ment plus élevé $(\mathrm{P}<0,05)$ chez les animaux castrés à 9 mois $(16,0 \%)$ que chez ceux castrés à 13 mois $(14,8 \%)$, quel que soit le niveau d'alimentation (tableau 3). Par ailleurs, le poids de carcasse des lots $\mathrm{HN}$ est plus élevé de $5 \mathrm{~kg}$ compte tenu du poids d'abattage supérieur, et les carcasses sont plus grasses $(+0,8 \%$ en dépôts adipeux rapportés au poids de carcasse).

\section{4 / Discussion}

Dans cette expérience, les animaux castrés tard n'ont conservé que peu d'avantage: à 20 mois en fin d'été, +5 à $6 \mathrm{~kg}$ selon la race; et avant le départ à l'abattoir, + $8 \mathrm{~kg}$ pour les charolais, + $5 \mathrm{~kg}$ pour les pie-noirs. En revanche, après l'abattage, le poids de carcasse a été inférieur à celui des animaux castrés à 9 mois pour les charolais ( $-6 \mathrm{~kg}$ ), ou identique pour les pienoirs $(+1 \mathrm{~kg})$. Toutefois la castration tardive a permis d'obtenir des carcasses moins grasses, du moins en race pie-noire où les mesures ont pu être réalisées.

Il est plus délicat de conclure à l'intérêt d'un haut niveau d'alimentation dans le jeune âge, d'autant qu'il n'a pas été appliqué à partir du même âge dans les deux races. Dans le cas des pie-noirs, le bas niveau entre 5 et 14 mois aurait pu être compensé par une durée de finition plus longue que celle appliquée de $31 \mathrm{j}$. Cela aurait pu permettre d'obtenir ainsi des carcasses de même poids que celles du lot HN, mais certainement plus grasses. Pour les deux races, le niveau d'alimentation élevé dans le jeune âge a permis d'accroître le gain de poids et le poids de carcasse des animaux castrés à 9 mois. Dans le cas des charolais, les meilleures performances finales sont atteintes par les $\mathrm{HN}$ castrés tôt et les BN castrés tard. On se serait plutôt attendu à ce que des apports alimentaires plus élevés vers l'âge d'un an soient favorables durablement aux animaux castrés tard, plus efficaces et sans doute restés plus maigres; et que le bas niveau soit mieux supporté par les animaux castrés tôt.

\section{3 / Effets de l'âge à la castration sur la production de boufs de deux ans (Essiai 3)}

Lors de la castration en début d'hiver $(270 \mathrm{j}$ d'âge), les 48 charolais pesaient en moyenne $316 \mathrm{~kg}$. Ils ont reçu pour moitié d'entre eux de l'ensilage d'herbe et pour l'autre de l'ensilage de maïs immature. La castration tardive a eu lieu 105 jours plus tard au poids moyen de $445 \mathrm{~kg}$.

\section{1 / Résultats}

Sur l'ensemble de l'hiver (20 semaines) les animaux restés entiers jusqu'à 13 mois ont ingéré seulement $3 \%$ d'herbe et $4 \%$ de maïs ensilés de plus que les animaux castrés à 9 mois (tableau 4). Rapportées au poids vif, ces consommations n'ont pas été influencées par l'âge à la castration, car les animaux castrés tard ont réalisé en moyenne des croîts plus élevés, $+115 \mathrm{~g} / \mathrm{j}$ soit $+12 \%$ (différence significative à $\mathrm{P}<0,05$ ).

Sur l'ensemble de l'hiver, les gains de poids vif des animaux recevant de l'ensilage de mais sont proches selon l'âge à la castration ; malgré des performances plus élevées au cours de la première partie de l'hiver, les animaux castrés à 13 mois ont mal supporté la castration en fin de période et atteignent des poids comparables à ceux des animaux castrés à 9 mois $(460 \mathrm{~kg}$ ). Le croît hivernal du lot recevant de l'ensilage d'herbe et castré tôt $(870 \mathrm{~g} / \mathrm{j})$ est très inférieur $(\mathrm{P}<0,05)$ à ceux des trois autres lots qui sont peu différents entre eux (1050 à $1080 \mathrm{~g} / \mathrm{j}$ environ).

A la mise à l'herbe, les animaux alimentés avec l'ensilage de maïs durant l'hiver se sont moins vite habitués à pâturer ; de plus, les animaux castrés 9 mois nourris avec l'ensilage d'herbe ont perdu beaucoup moins de poids à la transition (13 kg, contre 25 à $36 \mathrm{~kg}$ ). Les
Rapportées au poids vif, les consommations d'ensilage n'ont pas été influencées par l'âge à la castration, mais, avec l'ensilage d'herbe, les croissances hivernales des animaux castrés plus tôt ont été plus faibles.
Tableau 4. Poids vifs, croissances et consommations au cours du premier hiver selon la nature de l'ensilage et l'âge à la castration (Essai 3).

\begin{tabular}{|c|c|c|c|c|}
\hline Nature de l'ensilage & \multicolumn{2}{|c|}{ Herbe } & \multicolumn{2}{|c|}{ Maïs } \\
\hline Age à la castration (mois) & 9 & 13 & 9 & 13 \\
\hline $\begin{array}{l}\text { Période de } 9 \text { à } 14 \text { mois } \\
\text { Consommation d'ensilage (kg MS/j) } \\
\text { Consommation d'ensilage } \\
\text { (kg MS/100 kg de poids vif) } \\
\text { Poids vif à la castration }(\mathrm{kg}) \\
\text { Croissance journalière }(\mathrm{g} / \mathrm{j})(132 \mathrm{j}) \\
\text { Poids vif à la fin de l'hiver }(\mathrm{kg})\end{array}$ & $\begin{array}{c}5,19 \\
1,58 \\
317 \pm 26 \\
869 \mathrm{a} \pm 126 \\
431 \pm 35\end{array}$ & $\begin{array}{c}5,35 \\
1,58 \\
446 \pm 35 \\
1047 \mathrm{~b} \pm 106 \\
457 \pm 31\end{array}$ & $\begin{array}{c}5,57 \\
1,68 \\
315 \pm 25 \\
1084 b \pm 151 \\
458 \pm 27\end{array}$ & $\begin{array}{c}5,80 \\
1,69 \\
443 \pm 35 \\
1062 \mathrm{~b} \pm 144 \\
461 \pm 28\end{array}$ \\
\hline $\begin{array}{l}\text { Période de } 14 \text { à } 20 \text { mois } \\
\text { Poids vif après la mise à l'herbe }(\mathrm{kg}) \\
\text { Poids vif à } 20 \text { mois à la fin du pâturage }(\mathrm{kg})\end{array}$ & $\begin{array}{l}418 \pm 36 \\
566 \pm 36\end{array}$ & $\begin{array}{l}432 \pm 29 \\
546 \pm 26\end{array}$ & $\begin{array}{l}422 \pm 22 \\
568 \pm 34\end{array}$ & $\begin{array}{l}428 \pm 24 \\
558 \pm 30\end{array}$ \\
\hline
\end{tabular}

Les valeurs suivies de lettres différentes sont significativement différentes $(P<0,05)$. 
Tableau 5. Résultats d'engraissement et d'abattage selon la nature de l'ensilage consommé au cours du premier hiver et de l'âge à la castration (Essai 3).

\begin{tabular}{|l|c|c|c|c|}
\hline \multicolumn{1}{|c|}{ Nature de l'ensilage } & \multicolumn{2}{c|}{ Herbe } & \multicolumn{2}{c|}{ Maïs } \\
\hline Age à la castration (mois) & 9 & 13 & 9 & 13 \\
\hline Période de finition de 20 à 24 mois & & & & \\
Croissance journalière (g/j) & $939 \mathrm{a} \pm 269$ & $987 \mathrm{a} \pm 150$ & $923 \mathrm{a} \pm 247$ & $1033 \mathrm{a} \pm 148$ \\
Poids vif départ à l'abattoir $(\mathrm{kg})$ & $689 \pm 34$ & $\mathbf{6 7 7 \pm 3 3}$ & $691 \pm 39$ & $690 \pm 30$ \\
Abattage & & & & \\
$\begin{array}{l}\text { Poids de carcasse froide }(\mathrm{kg}) \\
\text { Dépôts adipeux en \% du poids } \\
\text { de carcasse }\end{array}$ & $420 \pm 26$ & $\mathbf{4 1 3 \pm 2 1}$ & $418 \pm 24$ & $410 \pm 12$ \\
& $17,4 \mathrm{a} \pm 3,1$ & $16,7 \mathrm{a} \pm 2,5$ & $17,0 \mathrm{a} \pm 2,0$ & $16,2 \mathrm{a} \pm 1,3$ \\
\hline
\end{tabular}

Les valeurs suivies de lettres différentes sont significativement différentes $(P<0,05)$.

poids des différents lots se sont donc fortement rapprochés en début de pâturage, quoique les animaux castrés à 13 mois aient alors gardé un avantage de $14 \mathrm{~kg}$ pour le lot herbe ensilée, de $6 \mathrm{~kg}$ pour le lot maïs ensilé (tableau 4).

Cet avantage a été perdu en cours de pâturage, car les animaux castrés à 9 mois ont réalisé $130 \mathrm{~g} / \mathrm{j}$ (soit $20 \%$ ) de plus que ceux castrés à 13 mois (différence significative, $\mathrm{P}<0,05$ ) (figure 3); et parmi ces derniers, les plus légers, qui avaient reçu l'herbe ensilée, ont eu une croissance supérieure de $80 \mathrm{~g} / \mathrm{j}$ par rapport au lot qui a reçu l'ensilage de maís en hiver. II en est résulté un effet de «ciseaux »: à 20 mois, les animaux castrés tôt sont les plus lourds, dépassant alors de $20 \mathrm{~kg}$ (lot ensilage d'herbe) et de $10 \mathrm{~kg}$ (lot ensilage de maïs) ceux castrés à 13 mois.

\section{2 / Finition et abattage à 24 mois}

Dans cet essai, les animaux alors âgés de 20 mois ont été transférés à l'INRA de Theix (63) et ont reçu une ration d'engraissement à base de maïs ensilé et de concentrés pendant 4 mois environ, jusqu'à l'abattage à deux ans (tableau 5).

Après transition, l'engraissement à l'auge a duré 102 j en moyenne. La croissance des animaux castrés à 13 mois a alors été de $80 \mathrm{~g} / \mathrm{j}$ (9\%) de plus que celle des animaux castrés 9 mois (différence non significative). La nature de l'ensilage distribué le premier hiver n'a pas eu d'effet sensible. Le poids moyen dans les quatre lots a été de $687 \mathrm{~kg}$ en fin d'expérience.

En définitive, sur l'ensemble de la durée expérimentale (9 à 24 mois), les gains totaux des animaux castrés à 9 mois ont été de $374 \mathrm{~kg}$ (372 et $376 \mathrm{~kg}$ respectivement avec l'ensilage d'herbe et de maïs en premier hiver) soit $10 \mathrm{~kg}$ de plus que ceux des animaux castrés à 13 mois (358 et $369 \mathrm{~kg}$ dans le même ordre). A l'issue de l'essai, c'est le lot recevant l'ensilage d'herbe et castré tard, qui avait le poids le plus élevé avant la castration, qui présente le gain total le plus faible.

A l'abattage (tableau 5), l'avantage des animaux castrés tôt est en moyenne de $7 \mathrm{~kg}$ de car- casse (moyenne des 4 lots $=415 \mathrm{~kg}$ ). Les mesures obtenues sur ces carcasses n'ont pas permis de mettre en évidence des états d'engraissement significativement différents; une tendance apparaît cependant avec des dépôts adipeux séparables moins importants dans le cas des animaux castrés à 13 mois $(16,5$ contre $17,2 \%$ de dépôts adipeux dans la carcasse).

\section{4 / Discussion - Conclusion}

Ce troisième essai n'a pu faire ressortir quelque avantage à la castration à 13 mois. Au

Figure 3. Evolution du poids vif des animaux entre 9 et 24 mois selon l'âge à la castration (essai 3).

poids vif $(\mathrm{kg})$

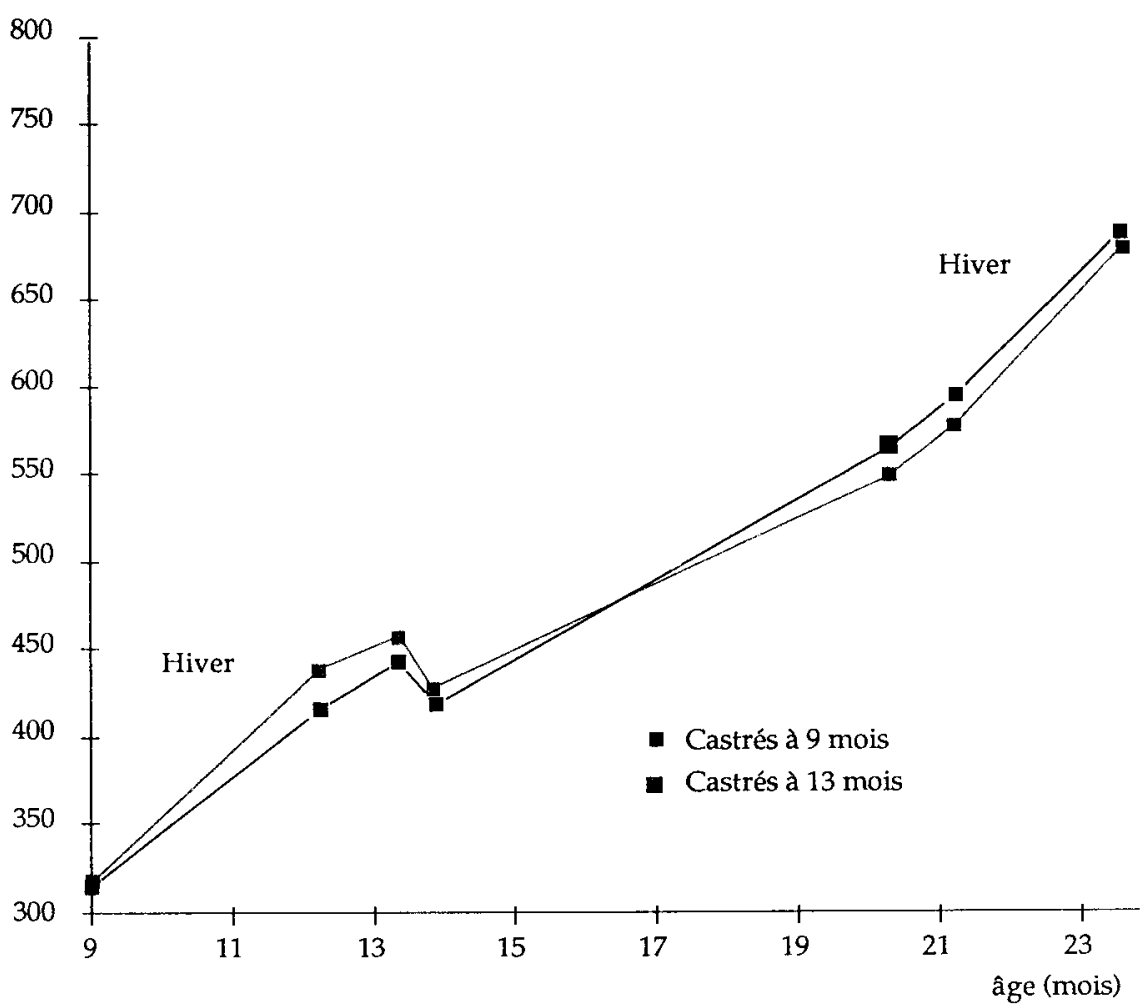

Les animaux castrés à 9 mois ont eu une croissance hivernale ralentie, mais ce retard a été compensé au cours de la période de pâturage. 
contraire, les animaux restés entiers jusqu'à cet âge ont semblé pénalisés pendant la période de pâturage par leur croît précédent plus élevé. Pendant l'engraissement, ils n'ont pu compenser tout le retard pris à 20 mois.

Or les essais 1 et 2 ont déjà mis en évidence cette compensation au pâturage en faveur des animaux castrés à 9 mois lorsque leurs croits avaient été plus faibles au cours du premier hiver ; de plus, les charolais castrés tard qui ont atteint les poids les plus élevés avant castration dans les essais 2 (lot haut niveau) et 3 (lot ensilage d'herbe) sont ceux qui ont fourni les poids de carcasse les plus faibles.

Inversement, la castration à 9 mois n'a pas désavantagé les animaux des races plus précoces que la charolaise, même lorsque leur croît a été durablement plus élevé dans le jeune âge (Pie-noirs en haut niveau de 5 à 14 mois : essai 2). Il ne semble donc pas qu'avec des animaux plus légers à 9 mois ou ayant des croîts moins élevés de 9 à 14 mois, la castration tardive puisse être avantageuse, quelle que soit la race, sur les performances globales de croissance. Rappelons en effet qu' en moyenne pour les 18 lots de 12 animaux utilisés, le poids lors de la castration précoce a été de $318 \mathrm{~kg}$ (extrêmes : 284 et $350 \mathrm{~kg}$ ) à un âge de $281 \mathrm{j}$, et de $436 \mathrm{~kg}$ (extrêmes : 389 et $482 \mathrm{~kg}$ ) à $388 \mathrm{j}$ d'âge lors de la castration à la fin de l'hiver. Le croît moyen des animaux restés entiers a donc été de l'ordre de $1100 \mathrm{~g}$ dans cet intervalle. Cependant chez les animaux de race précoce la castration à 9 mois semble se traduire à l'abattage par des carcasses présentant un état d'engraissement supérieur, de l'ordre de $8 \%$ (essai 2) pour des poids comparables.

L'ensemble de ces résultats converge avec ceux de la bibliographie obtenus sur des races très précoces (Angus, Hereford...). L'âge ou le poids à la castration, sur une large plage de variation, ne modifie pas significativement les performances globales de croissance (Worrell et al 1987). Cependant une castration tardive permet de réduire dans une faible mesure l'état d'engraissement final des animaux (Champagne et al 1969, Worrell et al 1987).

En définitive le choix de l'éleveur d'animaux de viande entre castration à la sortie du pré, ou en fin d'hivernage, sera plutôt déterminé par la pénibilité de l'opération, qui est aussi mieux supportée par l'animal plus jeune.

\section{Références bibliographiques}

CHAMPAGNE J.R., CARPENTIER J.W., HENTGES J.F., PALMER Jr. A.Z., KOGER M., 1969. Feedlot performance and carcass characteristics of young bulls and steers castrated at four ages. J. Anim. Sci. 29:887-890.

MICOL D., BERANGER C., 1984. French beef production systems from grassland. In : Grassland beef production. W. Holmes Ed., Martinus Nijhoff Publishers, Boston. p 11-22.

MICOL D., 1986. Production de boeufs et de jeunes taureaux. In : Production de viande bovine D. Micol Ed., INRA Publications, Paris. p 169-200.
NOCQUET J., 1977. Production de viande à partir de jeunes bovins abattus à deux ans ; intérêt de la castration, incidence du niveau alimentaire entre 20 et 24 mois. Mémoire de fin d'études ENSA Rennes, pp 21.

ROBELIN J., BOCCARD R., 1978. Effet de la castration tardive sur les qualités des viandes de différentes races. Ann. Tech. Agri. (27) p 565-584.

SEIDEMAN S.C., CROSS H.R., OLTJEN R.R., SCHANBACHER B.D., 1982. Utilization of the intact male for red meat production : a review. J. Anim. Sci. 55 : 826-840.

WORRELL M.A., CLANTON D.C., CALKINS C.R., 1987. Effect of weight at castration on steer performance in the feedlot. J. Anim. Sci. $64: 343-347$

\section{Summary}

Choice of age of castration in grass beef production.

Beef production from grass in France is based on castrated animals. However the uncastrated male has a faster growth rate and presences leaner meat. This had led to the suggestion that young bulls could be castrated later with an increased risk of stress and disease if the animals have already reached a certain stage of development.

The aim of the studies carried out in Le Pin au Haras INRA experimental farm was to understand the effects of age at castration ( 9 or 13 months] on grass beef production. These effects have been studied in relation to others factors during three trials : maturity of breeds, feeding level during the rearing period and slaughter age.

It appears that the age at castration does not modify appreciably animal characteristics at slaughter. Many interactions during the rearing period and compensatory growth effects reduce the expected differences with castration age. However the carcases of late castrated animals seem to be leaner.

Finally under working conditions the choice of age of castration (around 1 year old) seems to be defined by practicality and risk of stress for the animal.

MULLER A., MICOL D., PECCA'TTE J.R., DOZIAS D., 1991. Choix de l'âge à la castration en production de viande bovine semi-intensive. INRA Prod. Anim., 4 (4), $287-295$. 
Annexe. Conduite des différents essais.

\begin{tabular}{|c|c|c|c|}
\hline Essai & 1 & 2 & 3 \\
\hline Dispositif & $\begin{array}{l}3 \text { génotypes } \\
2 \text { âges à la } \\
\text { castration }\end{array}$ & $\begin{array}{l}2 \text { génotypes } \\
2 \text { âges à la } \\
\text { castration } \\
2 \text { niveaux } \\
\text { d'alimentation }\end{array}$ & $\begin{array}{l}1 \text { génotype } \\
2 \text { âges à la } \\
\text { castration } \\
2 \text { types } \\
\text { d'ensilage }\end{array}$ \\
\hline Animaux & $\begin{array}{l}24 \text { charolais } \\
24 \text { normands } \\
26 \text { pie-noirs }\end{array}$ & $\begin{array}{l}48 \text { charolais } \\
48 \text { pie-noirs }\end{array}$ & 48 charolais \\
\hline $\begin{array}{l}\text { Ration } \\
1^{\text {er }} \text { hiver }\end{array}$ & $\begin{array}{l}\text { Ensilage de maïs } \\
(23 \% \mathrm{MS}) \\
+1 \mathrm{~kg} \text { de tourteau } \\
\text { puis } \\
\text { Ray grass anglais } \\
(21 \% \mathrm{MS}) \\
+0,5 \mathrm{~kg} \text { d'orge }\end{array}$ & $\begin{array}{l}\text { Ensilage de maïs } \\
\text { (25\% MS }) \\
+0,5 \mathrm{~kg} \text { de tourteau } \\
\text { puis } \\
\text { Ray grass anglais } \\
(19 \% \mathrm{MS}) \\
+0,5 \mathrm{~kg} \text { de tourteau }\end{array}$ & $\begin{array}{l}\text { Ensilage de maïs } \\
(21 \% \mathrm{MS}) \\
+1 \mathrm{~kg} \text { de tourteau } \\
\text { puis } \\
\text { Ray grass anglais } \\
(20 \% \mathrm{MS}) \\
+1 \mathrm{~kg} \text { concentré }\end{array}$ \\
\hline $\begin{array}{l}\text { Niveau } \\
\text { d'alimentation } \\
1^{\text {er hiver }}\end{array}$ & - & $\begin{array}{l}\text { Bas : } \\
0 \text { kg de concentré } \\
\text { Haut : } \\
1,5 \mathrm{~kg} \text { de concentré }\end{array}$ & - \\
\hline $2^{\mathrm{e}}$ été & \multicolumn{3}{|c|}{ Pâturage dans les 3 essais } \\
\hline $\begin{array}{l}\text { Ration } \\
2^{\mathrm{e}} \text { hiver }\end{array}$ & Non expérimental & $\begin{array}{l}\text { Fourrages } \\
\text { médiocres }\end{array}$ & $\begin{array}{l}\text { Maïs ensilé } \\
+ \text { concentrés }\end{array}$ \\
\hline Finition & $\begin{array}{l}\text { Au pâturage } \\
\text { à } 30 \text { mois }\end{array}$ & $\begin{array}{l}\text { Au pâturage } \\
\text { à } 30-32 \text { mois }\end{array}$ & $\begin{array}{l}\text { A l'auge } \\
\text { à } 24 \text { mois }\end{array}$ \\
\hline
\end{tabular}

